\title{
Human neural progenitor cells display limited cytotoxicity and increased oligodendrogenesis during inflammation
}

\author{
Cell Death and Differentiation (2007) 14, 876-878. doi:10.1038/sj.cdd.4402078; published online 22 December 2006
}

Dear Editor,

The ability to cultivate and expand neural stem and progenitor cells introduced a new therapeutic prospective for the treatment of neurodegenerative conditions, such as Parkinson disease, stroke, Alzheimer disease and multiple sclerosis. ${ }^{1-3}$ The inflammatory environment surrounding the injured areas during brain damage can be deleterious for the survival of neurons and oligodendrocytes. In contrast, endogenous neural progenitor cells (NPCs) are attracted to the site of lesion where they survive and proliferate, perhaps as part of a restoring mechanism. ${ }^{4}$ We have demonstrated that the ability of NPCs to survive in the context of unfavorable microenvironment results from the absence of caspase 8 and the overexpression of the antiapoptotic protein PED/PEA-15. Such a double level of protection is most likely responsible for the survival of NPCs during brain damage, which is often characterized by the presence of proinflammatory cytokines and death receptor ligands.

The use of reliable preclinical models of neurodegenerative diseases is a mandatory step for the understanding of the mechanisms responsible for disease onset and progression, as well as for establishing new and effective therapies. Pluchino et al. ${ }^{6}$ recently reported that upon exposure to proinflammatory cytokines, mouse NPCs express CD95 ligand (CD95L), TRAIL and TWEAK, becoming able to kill surrounding activated $\mathrm{T}$ cells. Although it is unlikely that TWEAK production could have any beneficial impact on the outcome of the autoimmune aggression, ${ }^{7,8}$ the ability of mouse neural stem and progenitor cells to kill activated T cells through the production of TRAIL and CD95L can be exploited for the treatment of experimental autoimmune encephalomyelitis (EAE), a mouse model of multiple sclerosis. In this system, the same authors showed that the systemic injection of mouse adult NPCs promoted neuroprotection and myelin regeneration by inducing apoptosis of encephalitogenic $\mathrm{T}$ cells. ${ }^{6}$ Thus, the cytotoxic activity of primitive mouse neural cells could enforce the therapeutic potential of experimental cellular therapies based on the use of mouse neurospheres to replenish the oligodendrocyte population killed during the autoimmune process.

The possible exploitation of the immunomodulatory activity of NPCs to treat autoimmune demyelination is very promising. However, these studies have been carried out only in the mouse. An extensive functional analysis of human neural stem cells and their progeny is therefore required before proposing the use of NPCs for the treatment of multiple sclerosis in the clinical setting.
Human stem and progenitor cells can be cultured in vitro as neurospheres in serum-free medium containing epidermal growth factor and basic fibroblast growth factor. ${ }^{9-13}$ In order to evaluate the effect of acute stimulation with inflammatory cytokines on NPCs, we exposed neurospheres at early passages to TNF- $\alpha, \mathrm{IL}-1 \beta$ and IFN- $\gamma$ and analyzed the expression of 94 apoptosis-related mRNA by a custom RTPCR array (Microfluidic Card, Applied Biosystems). Interestingly, we observed that in the presence of proinflammatory cytokines nestin-positive primitive neural cells express considerable amounts of TNF- $\alpha$, TL1A and TRAIL mRNA, while showing no detectable CD95L (data not shown). These results were confirmed by real-time $\mathrm{PCR}$, using commercial ready-to-use primers/probe mixes (Figure 1a). The expression of these death receptor ligands was subsequently confirmed at the protein level by immunoblot analysis, which revealed that proinflammatory cytokines induced a considerable increase in TL1A and TNF- $\alpha$, whereas TRAIL expression almost doubled within $12 \mathrm{~h}$ but remained stable afterwards (Figure 1b), despite the massive increase observed at the mRNA level.

The absence of CD95L questioned the ability of human NPCs to kill activated T cells, whereas the expression of high levels of TNF- $\alpha$ suggested a higher cytotoxic potential against monocytic-macrophagic cells. To evaluate the cytotoxic activity of human NPCs, we primed human neurospheres for $60 \mathrm{~h}$ with proinflammatory cytokines before carrying out co-culture experiments with human monocytic (U937) and lymphocytic (Jurkat) cell lines pre-labeled with Oregon Green 488 long-term cell tracer (Molecular Probes). After overnight incubation, cells were stained with Alexa 647-conjugated Annexin V (Molecular Probes) and propidium iodide (Sigma Aldrich) to evaluate the number of apoptotic target cells. Flow cytometry analysis was performed by gating on Oregon Green labeled cells to identify target cells and exclude NPCs. This procedure allowed an accurate quantification of the cytotoxic activity of NPCs towards hematopoietic target cells (Figure 1c). In line with the absence of CD95L and with the high production of TNF- $\alpha$, such a cytotoxic activity was weak on lymphocytic cells and considerably higher on monocytic cells (Figure 1d).

To verify that the cytotoxic activity of NPCs was specifically due to DR engagement, we transduced U937 cells with a lentiviral vector carrying both the cDNA of the green fluorescent protein (GFP) reporter and the antiapoptotic protein c-Flip, which counteracts the activation of caspase 8 

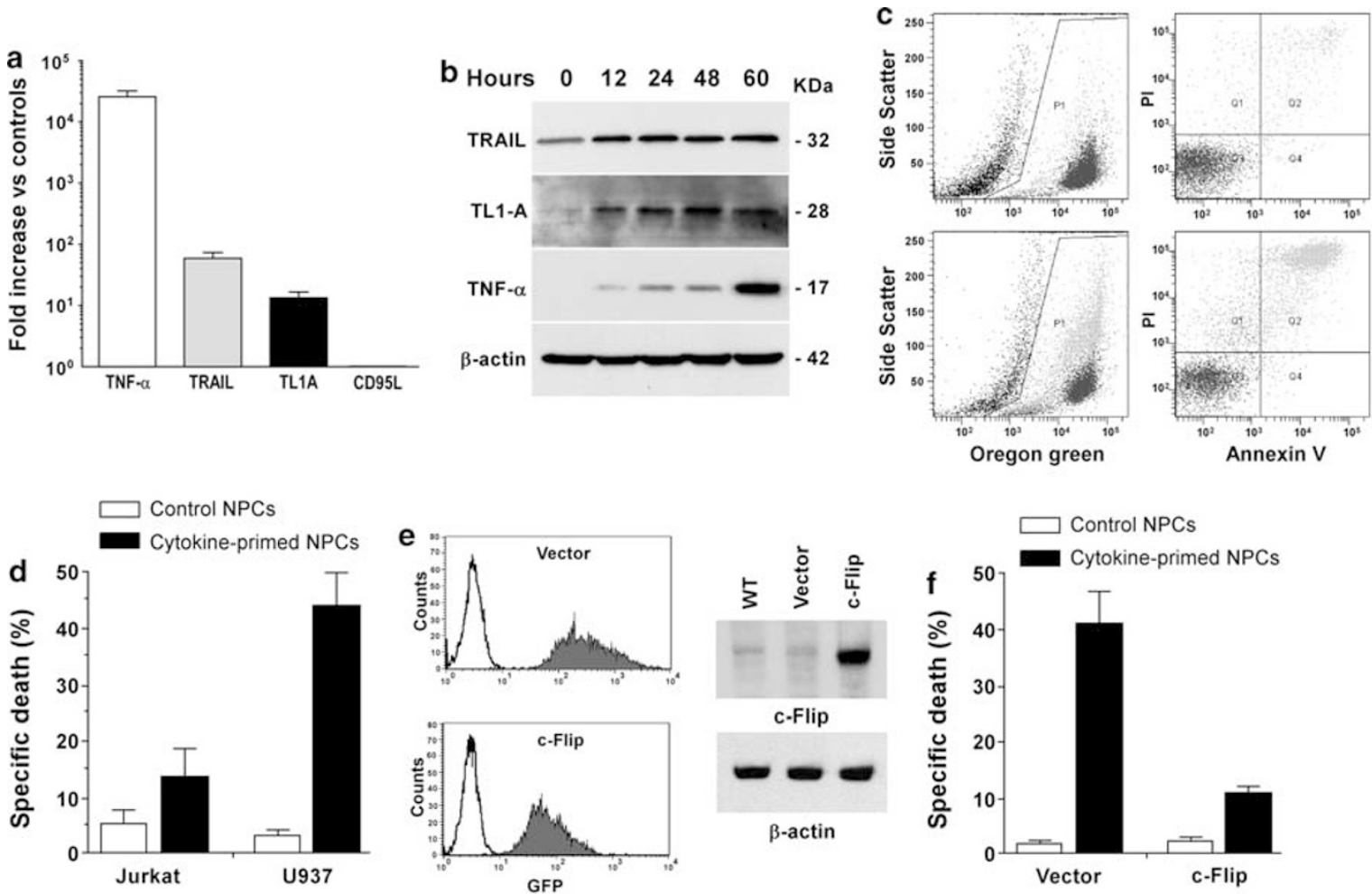

C-Flip

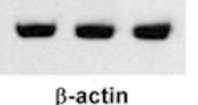

$\beta$-actin

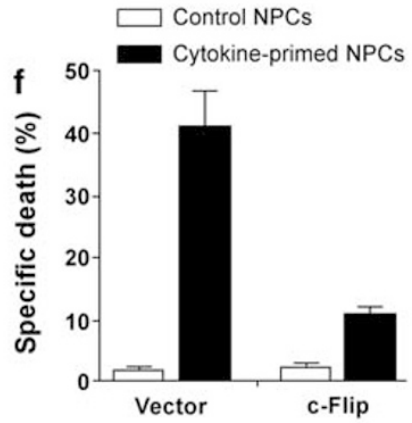

g

Neutralized TL1A TL1A TRAIL TRF- $\alpha$

ligand $\longrightarrow$ TL1A TRAIL TNF- $\alpha$ TRAIL TNF- $\alpha$ TNF- $\alpha$ TL1A
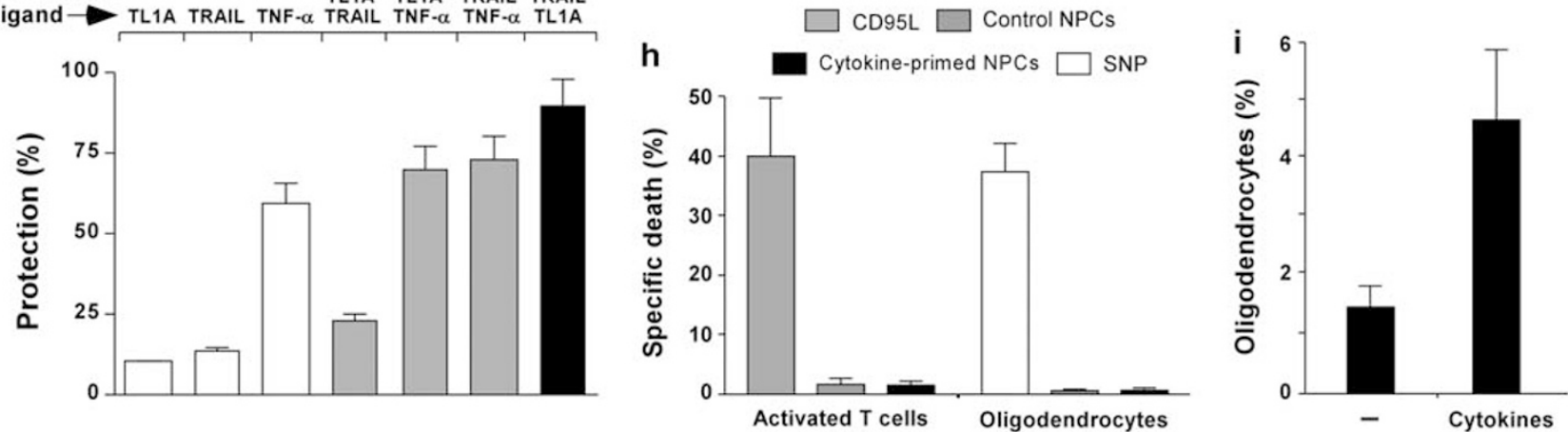

Figure 1 Cytotoxic activity of NPCs exposed to proinflammatory cytokines. (a) mRNA and (b) protein levels of death receptor ligands as detected by real-time PCR and Western blotting, respectively. Cells were incubated with TNF- $\alpha 200 \mathrm{U} / \mathrm{ml}$, IFN- $\gamma 500 \mathrm{U} / \mathrm{ml}$ and IL1- $\beta 100 \mathrm{U} / \mathrm{ml}$, and then lysed for RNA and protein extraction. Real-time PCR values are mean \pm S.D. of mRNA levels, normalized on untreated samples, of two embryonic and one adult neurosphere cell lines exposed for $60 \mathrm{~h}$ to proinflammatory cytokines. (c) U937 cells were labeled with Oregon Green long-term cell tracer (Molecular Probes) and then co-cultured for $16 \mathrm{~h}$ with NPCs, untreated (top) or previously exposed to inflammatory cytokines for $60 \mathrm{~h}$ (bottom). Target cell viability was analyzed by flow cytometry through the evaluation of Annexin-V (AlexaFluor 647 conjugated) and propidium iodide staining on Oregon Green positive cells. (d) Jurkat and U937 cell lines co-cultured with NPCs untreated (Control NPCs) or previously exposed to inflammatory cytokines for $60 \mathrm{~h}$ (Cytokine-primed NPCs). Target cells were stained as in c and percentage of specific death was calculated. Data are expressed as mean \pm S.D. of 10 independent experiments with three embryonic and one adult effector cell lines. (e) Flow cytometry profiles of GFPpositive U937 (grey) transduced with empty Tween (Vector) or Tween/c-Flip (c-Flip) as compared with untransduced (white) U937 (left panel). Immunoblot analysis of cFlip and $\beta$-actin in cells transduced as above (right panel). (f) Specific cytotoxicity of NPCs against empty vector and c-Flip-transduced U937 cells as shown in (e). (g) Effect of single or multiple death receptor ligand neutralization by DR3-Fc, DR4-Fc and anti-TNF- $\alpha$ on cytokine-primed NPC toxicity against U937 cells. Data are expressed as mean \pm S.D. of three independent experiments with two embryonic and one adult effector cell lines. Percentage of protection was determined as $100-X$, where $X$ was calculated with the following formula: $(C 1-C 2): 100=(S 1-S 2): X . C$ states for control and S states for sample, 1 refers the viability of untreated cells and 2 to the viability of cells treated with the apoptotic stimulus. (h) Human activated T cells and differentiated neural cells were stained with Oregon green as above and exposed for $24 \mathrm{~h}$ to different potential cytotoxic stimuli as indicated. Recombinant CD95L $(200 \mathrm{ng} / \mathrm{ml})$ and $1 \mathrm{mM}$ sodium nitroprusside (SNP) were used as positive controls. (i) Evaluation of serum-induced oligodendrocytic differentiation of NPCs previously exposed to proinflammatory cytokines for $60 \mathrm{~h}$ as above. The number of oligodendrocytes was calculated based on the percentage of GalC positive cells

after death receptor stimulation. ${ }^{14}$ The infections were monitored by flow cytometry until we obtained a virtually pure population of transduced cells expressing GFP alone (Vector) or in combination with c-Flip, whose expression was readily detectable by immunoblot analysis (Figure 1e). As expected, c-Flip expression in U937 cells greatly reduced the killing 
activity of cytokine-primed NPCs (Figure 1f), confirming the prevalent involvement of death receptors in target cell apoptosis.

To evaluate the role of single death receptor ligands in NPC-mediated cytotoxicity, we performed the co-culture experiments in the presence of blocking antibodies or soluble receptors specific for each ligand. The blocking agents were used either individually or in combination. This experiment revealed that TRAIL and TL1A contribute marginally to the cytotoxic activity of cytokine-primed human NPCs, which was mainly mediated by TNF- $\alpha$, whose neutralization restored the survival of more than $50 \%$ of the target cells killed by NPCs (Figure 1g). However, the three death ligands displayed additive cytotoxic activity, as demonstrated by the observation that the highest protection was obtained after the simultaneous neutralization of TNF- $\alpha$, TRAIL and TL1A (Figure 1g). Despite the ability of TL1A to induce apoptosis through the engagement of DR3 in some cell types, this ligand is a survival factor for $T$ cells and promotes their expansion during the immune response. ${ }^{15}$ Thus, the absence of CD95L and the considerable induction of TL1A in human NPCs during inflammation is unlikely to result in the massive T-cell apoptosis reported in the mouse. Accordingly, we observed that cytokine-primed NPCs did not show any cytotoxic activity on activated $\mathrm{T}$ cells (Figure $1 \mathrm{~h}$ ), indicating that the absence of CD95L expression of human NPCs dramatically reduced their cytotoxic potential towards T cells. Although TNF receptor II can promote oligodendrocyte regeneration in neurotoxical models of demyelinization, ${ }^{16}$ TNF- $\alpha$ has been hypothesized to directly contribute to autoimmune oligodendrocyte destruction through the engagement of TNF receptor I. ${ }^{17}$ However, we did not detect any cytotoxic activity of cytokine-exposed NPCs on human oligodendrocytes (Figure 1h). Moreover, we observed that the exposure to proinflammatory cytokines increased oligodendrocyte generation during serum-induced differentiation of NPCs (Figure 1i), suggesting that multipotent NPCs may increase their myelinotrophic activity during inflammation.

Taken together, our data indicate that different from the mouse counterparts, human NPCs display a limited cytotoxicity towards $T$ cells, while being more active on monocytic cells through the TNF pathway. Although autoreactive T cells may be sensitive to TRAIL, the considerable increase in TRAIL mRNA observed in cytokine-primed NPCs is not paralleled by an equal increase at protein level, in line with the weak TRAIL-mediated cytotoxicity shown by human NPCs on U937 cells. Despite the reduced immunomodulatory activity of human NPCs on T cells, these cells seem to have a higher capacity of generating oligodendrocytic cells in inflammatory conditions. This feature is in line with the possible direct contribution of donor NPCs to myelin reensheathment observed in EAE mice. ${ }^{18}$

Although human NPCs may be unable to reduce significantly the autoimmune response, resistance to apoptosis and increased oligodendrocyte formation during inflammation seem compatible with a possible future therapeutic transplantation of NPCs for the treatment of multiple sclerosis. In this view, it will be critical to confirm in humans the considerable migratory capacity of mouse NPCs, which can cross the blood-brain barrier and localize in the demyelinated areas. ${ }^{18}$

In conclusion, although further experiments are required to validate the possible therapeutic use of human NPCs for the treatment of neurological disorders, preclinical data should take into account species differences that might alter the outcome of the treatment.

Acknowledgements. This work was supported by grants from Associazione Italiana per la Ricerca sul Cancro the Ministero della Salute and the Ministero dell'Università e della Ricerca Scientifica e Tecnologica (Project No RBIP06ZJ78) to RDM.

\section{Ricci-Vitiani $^{1}$, DG Lombardi ${ }^{2}$, M Signore ${ }^{1}$, M Biffoni $^{1}$,

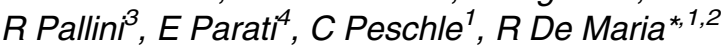

${ }^{1}$ Department of Hematology, Oncology and Molecular Medicine, Istituto Superiore di Sanità, Rome, Italy

2 Department of Experimental Oncology, Mediterranean Institute of Oncology, Catania, Italy

${ }^{3}$ Department of Neurosurgery, Catholic University, Rome, Italy

${ }^{4}$ Laboratory of Neurobiology, Department of Neurobiology and Neurorestorative Therapies, National Neurological Institute'C. Besta', Milan, Italy

* Corresponding author: R De Maria, Department of Hematology, Oncology and Molecular Medicine, Istituto Superiore di Sanità, viale Regina Elena 299, Rome 00161, Italy. Tel: + 39 0649903121; Fax: + 39 0649387087;

E-mail: rdemaria@tin.it

1. Gage FH. Science 2000; 287 : 1433-1438.

2. Reynolds BA, Weiss S. Science 1992; 255: 1707-1710.

3. Rossi F, Cattaneo E. Nat Rev Neurosci 2002; 3: 401-409.

4. Kokaia Z, Lindvall O. Curr Opin Neurobiol 2003; 13: 127-132.

5. Ricci-Vitiani L et al. J Exp Med 2004; 200: 1257-1266.

6. Pluchino $S$ et al. Nature 2005; 436: 266-271.

7. Desplat-Jego $S$ et al. J Neuroimmunol 2002; 133: 116-123.

8. Desplat-Jego $S$ et al. Clin Immunol 2005; 117: 15-23.

9. Caldwell MA et al. Nat Biotechnol 2001; 19: 475-479.

10. Vescovi AL et al. Exp Neurol 1999; 156: 71-83.

11. Galli R et al. Dev Neurosci 2000; 22: 86-95.

12. Pagano SF et al. Stem Cells 2000; 18: 295-300.

13. Reynolds BA, Rietze RL. Nat Methods 2005; 2: 333-336.

14. Irmler M et al. Nature 1997; 388: 190-195.

15. Migone TS et al. Immunity 2002; 16: 479-492.

16. Arnett HA et al. Nat Neurosci 2001; 4: 1116-1122.

17. Hovelmeyer $\mathrm{N}$ et al. J Immunol 2005; 175: 5875-5884.

18. Pluchino $S$ et al. Nature 2003; 422: 688-694. 